\title{
Appendical Perforation by Infection with Extended-Spectrum Beta-Lactamase (ESBL)-Producing Escherichia coli: Case Report
}

\author{
Hisayuki Miyagi ${ }^{1}$, Tadao Okada ${ }^{1 *}$, Shohei Honda ${ }^{1}$, Masashi Minato ${ }^{1}$, \\ Akinobu Taketomi ${ }^{2}$ \\ ${ }^{1}$ Department of Pediatric Surgery, Hokkaido University Hospital, Sapporo, Japan \\ ${ }^{2}$ Department of Gastroenterological Surgery I, Hokkaido University Graduate School of Medicine, \\ Sapporo, Japan \\ Email: *okadata@med.hokudai.ac.jp
}

Received September 25, 2011; revised November 30, 2011; accepted December 9, 2011

\begin{abstract}
We report the very rare case of a huge appendical abscess with extended-spectrum beta-lactamase (ESBL)-producing Escherichia coli (E. coli) as the pathogen. There have been several reports of appendical infections such as appendicitis and appendical abscess caused by ESBL-producing bacteria in adults. The treatment of ESBL-producing E. coli infection is specific, and ESBL-producing bacteria have recently been reported as pathogens associated appendicitis in children. To the best of our knowledge, this is the second report of perforated appendicitis with abscess due to ESBL-producing E. coli. We discuss the diagnostic modalities and treatments for appendical abscess with ESBL-producing E. coli. and propose that the patients with perforated appendicitis and abscess formation due to ESBL-producing E. coli should be administered the antibiotic MEPM within 2 weeks to treat the abscess more effectively without producing other multidrug-resistant bacteria.
\end{abstract}

Keywords: Appendicitis; ESBL (Extended-Spectrum Beta-Lactamase)-Producing E. coli; Abscess; Meropenem

\section{Introduction}

Beta-lactamases open the beta lactam ring of antibiotics and confer resistance to antibiotics. In the 1960s and 70s, beta-lactamases conferred resistance to penicillins and narrow-spectrum cephalosporins, such as cephalothin or cefazolin. The second- and third-generation cephalosporins, with an oxyimino side chain such as cefotaxime (CTX), ceftazidime (CAZ), ceftriaxone (CTRX), and cefepim (CFPM), were effective against beta-lactamaseproducing bacteria at that time [1]. Since the late 90s, the prevalence of extended-spectrum beta-lactamase (ESBL)producing bacteria has been increasing throughout the world $[2,3]$. In Japan, year on year, the rate of ESBLproducing bacteria has been rising [4]. Infections due to ESBL-producing bacteria will critically affect ill patients because of their resistance to many of the antimicrobial agents.

We review the literature on this type disorder, and discuss the problems associated with the characteristics of ESBL-producing E. coli, particular concerning the diagnostic and surgical modalities for appendical abscess

\footnotetext{
${ }^{*}$ Corresponding author.
}

complicated by the ESBL-producing E. coli.

\section{Case Report}

A 12-year-old girl was referred to our hospital with a marked fever and right lower abdominal pain after the administration of a third-generation cephalosporin, CTRX, for 4 days. Laboratory examination showed high levels of inflammatory markers: white blood cells,

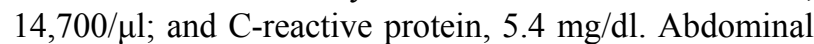
ultrasonography and enhanced computed tomography revealed a huge abscess, $7.8 \times 4.1 \times 6.8 \mathrm{~cm}$ in diameter, behind the cecum (Figures 1(a) and (b)). Based on these findings, the patient was diagnosed with appendical abscess, and underwent emergent surgery on the same day. On laparotomy, appendectomy and drainage with closed suction for abscess were performed. The histopathological finding of the resected appendix was gangrenous appendicitis, and ESBL-producing E. coli was positive on intra-operative culture of the abscess. The antibiotic sensitivity for ESBL-producing E. coli was positive only for carbapenem (e.g., MEPM). Therefore, we changed the antibiotics from cefmetazole (CMZ) to MEPM to treat the abscess more effectively. After 2 weeks of admini- 


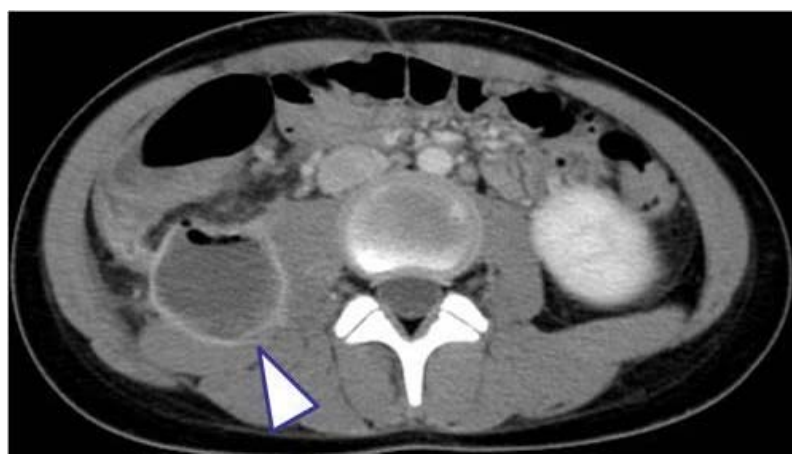

(a)

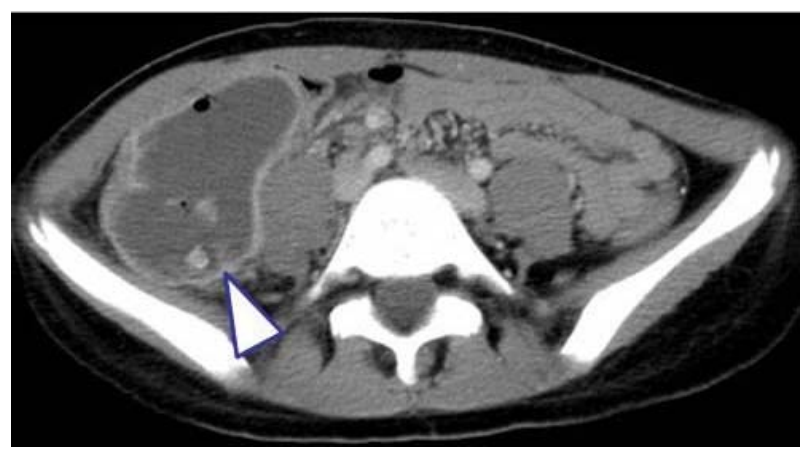

(b)

Figure 1. CT scan showing a huge abscess behind the cecum. stration of MEPM, drainage culture of the abscess was negative for 3 times, and the patient was discharged on the 23rd post-operative day (Figure 2). After 6 months of follow-up, there were no signs of recurrence of the abscess.

\section{Discussion}

ESBL-producing bacteria are viewed as producing new beta-lactamases, although they were first described in Germany as long ago as 1983 [5]. They are able to hydrolyse the oxymino group, thus conferring resistance to most beta-lactam antibiotics, except for carbapenems (e.g., MEPM) and cephamycins. They are often encoded in large plasmids, which means that additional resistance mutation can be carried by the same organism, including those leading to resistance to aminoglycosides and quinolones. Thus, many of these organisms are multi-drugresistant, and therapeutic options using antimicrobial agents are limited [6].

Currently, ESBL-producing bacteria are an increasingly common cause of human infection. These organisms are frequently resistant to many of the antimicrobial agents used to treat infections with gram-negative bacteria. $E$. coli is now one of the main gram-negative species to cause infections with ESBL-positive bacteria in humans [7]. Hague R. reported that no child-specific data

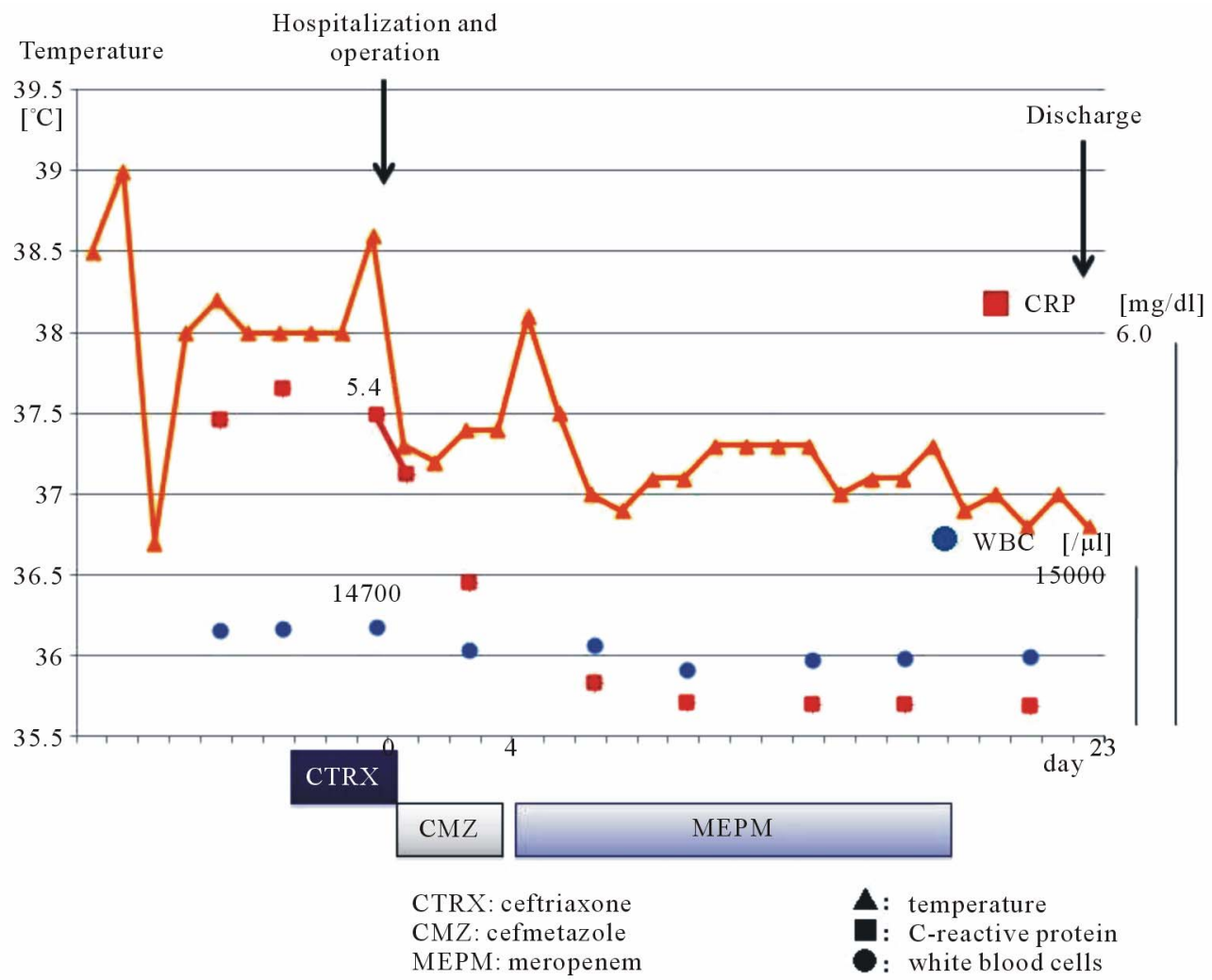

Figure 2. Clinical course of the patient. 
had been published for the UK, but overall rates of resistance to third-generation cephalosporins in E. coli isolates causing systemic infection rose significantly from around $2 \%$ in 2000 to $12 \%$ in 2007 [6]. In Japan, year on year, the rate of ESBL-producing bacteria has also been rising [4]. To the best of our knowledge, this is the second report [8] of perforated appendicitis complicated with abscess due to ESBL-producing E. coli.

According to the options of surgical treatment for appendical abscess due to ESBL-producing E. coli, a primary operation with appendectomy and abscess drainage and delayed appendectomy are selected.

In the present case, the appendical abscess was huge and of the retrocecal type. Therefore, we performed the primary operation in consideration of a short hospital stay and hesitation to avoid the long administration of antimicrobial agents.

In summary, we propose that patients with perforated appendicitis and abscess formation due to ESBL-producing E. coli should be administered the antibiotic MEPM within 2 weeks to treat the abscess more effectively without producing other multidrug-resistant bacteria.

\section{REFERENCES}

[1] S. Ito, H. Machida, T. Harada, et al., "Extended-Spectrum Beta-Lactamases-Producing Bacteria and Vesicoureteral Reflux in Children," Pediatrics International, Vol. 52, No. 1, 2010, pp. 134-137. doi:10.1111/j.1442-200X.2009.03011.x

[2] D. L. Paterson and R. A. Bonomo, "Extended-Spectrum Beta-Lactamases: A Clinical Update," Clinical Microbiology Reviews, Vol. 18, No. 4, 2005, pp. 657-686.

\section{doi:10.1128/CMR.18.4.657-686.2005}

[3] P. L. Winokur, R. Canton, J. M. Casellas, et al., "Variations in the Prevalence of Strains Expressing an Extended-Spectrum Beta-Lactamase Phenotype and Characterization of isolates from Europe, the Americas, and the Western Pacific Region," Clinical Infectious Diseases, Vol. 32, Suppl. 2, 2001, pp. 94-103. doi: $10.1086 / 320182$

[4] K. Yamaguchi and A. Ohno, "Investigation of the Susceptibility Trends in Japan to Fluoroquinolones and Other Antimicrobial Agents in a Nationwide Collection of Clinical Isolates: A Longitudinal Analysis from 1994 to 2002," Diagnostic Microbiology and Infectious Disease, Vol. 52, No. 2, 2005, pp. 35-43. doi:10.1016/j.diagmicrobio.2005.02.009

[5] H. Knothe, P. Shah, V. Krcmery, et al., "Transferable Resistance to Cefotaxime, Cefoxitin, Cefamandole and Cefuroxime in Clinical Isolates of Klebsiella Pneumoniae and Serratia Marcescens," Infection, Vol. 11, No. 6, 1983, pp. 315-317. doi:10.1007/BF01641355

[6] R. Hague, "What is the Threat from Extended Spectrum Beta-Lactamase-Producing Organisms in Children?" Archives of Disease in Childhood, Vol. 96, 2011, pp. 325327. doi:10.1136/adc.2010.184911

[7] T. E. Peter, G. Deepakraj, J. Brian, et al., "Transmission of an Extended-Spectrum-Beta-Lactamase-Producing Escherichia coli (Sequence Type ST131) Strain between a Father and Daughter Resulting in Septic Shock and Emphysematous Pyelonephritis," Journal of Clinical Microbiology, Vol. 47, No. 11, 2009, pp. 3780-3782. doi:10.1128/JCM.01361-09

[8] M. Nakata, Y. Miyauchi, M. Sonoda, et al., "A Case of Appendicitis with Postoperative Infections Due to ESBLProducing E. coli," Japanese Journal of Pediatric Surgery, Vol. 41, 2009, pp. 762-766. 